\title{
The role of transient attention in crowding and feature binding
}

\author{
Bahiyya Kewan-Khalayly ${ }^{1}$ \& Amit Yashar $^{1}$
}

1The Department of Special Education, The Edmond J. Safra Brain Research Center for the Study of Learning Disabilities, The University of Haifa, Israel

Email: amit.yashar@edu.haifa.ac.il

URL: https://yasharlab.com

Corresponding author:

Amit Yashar

amit.yashar@edu.haifa.ac.il

Faculty of Education

University of Haifa, 199 Abba Khoushy Ave, Haifa, 3498838, Israel

Phone: +972 (4) 8249297

Funding:

This work was supported by The Israel Science Foundation Grant Nos. 1980/18 (to A. Yashar).

Competing interests:

The authors have declared that no commercial conflict of interest exist. 


\title{
ATTENTION ASYMMETRY
}

\begin{abstract}
Crowding refers to the failure to identify a peripheral object due to nearby objects (flankers). A hallmark of crowding is inner-outer asymmetry; i.e., the outer flanker (more peripheral) produces stronger interference than the inner one. Here, by manipulating attention, we tested the predictions of two competing accounts: the attentional account, which predicts a positive attentional effect on the inner-outer asymmetry (i.e., attention to the outer flanker will increase asymmetry) and the receptive field size account, which predicts a negative attentional effect. In Experiment 1, observers estimated a Gabor target orientation. A peripheral pre-cue drew attention to one of three locations: target, inner flanker or outer flanker. Probabilistic mixture modeling demonstrated asymmetry by showing that observers often misreported the outerflanker orientation as the target. Interestingly, the outer cue led to a higher misreport rate of the outer flanker, and the inner cue led to a lower misreport rate of the outer flanker. Experiment 2 tested the effect of crowding and attention on incoherent object reports (i.e., binding errors - reporting the tilt of one presented item with the color of another item). In each trial, observers estimated both the tilt and color of the target. Attention merely increased coherent target reports, but not coherent flanker reports. The results suggest that the locus of spatial attention plays an essential role in crowding as well as inner-outer asymmetry and demonstrate that crowding and feature binding are closely related. However, our findings are inconsistent with the view that covert attention automatically binds features together.
\end{abstract}

Keywords: crowding; attention; binding; mixture model; asymmetry; color; orientation 
In vision, objects' spacing can fundamentally limit object recognition. Objects that are too close together can become indistinguishable (cluttered), a phenomenon known as 'crowding' (Pelli, 2008; Whitney \& Levi, 2011). Crowding hinders the identification of various basic stimuli, such as letters (Bouma, 1970) and faces (Strasburger, Rentschler, \& Jüttner, 2011), and impairs essential perceptual tasks such as reading (Whitney \& Levi, 2011) and face recognition (Strasburger et al., 2011). Crowding plays a critical role in deficits such as macular degeneration (Wallace, Chung, \& Tjan, 2017), amblyopia (Song, Levi, \& Pelli, 2014) and dyslexia (Gori \& Facoetti, 2015). Thus, investigating crowding and the means to reduce its disruptive effect has important implications for understanding object recognition and has the potential for clinical contribution (Levi, 2008).

The critical spacing of crowding - that is, the minimal space between the target and the flankers that permits performance similar to when no flankers are presented-scales with target eccentricity (Bouma, 1970; Pelli, Palomares, \& Majaj, 2004). Although spatial attention (Yeshurun \& Rashal, 2010) and training (perceptual learning) (Chung, 2007; Hussain, Webb, Astle, \& McGraw, 2012; Yashar, Chen, \& Carrasco, 2015; Zhu, Fan, \& Fang, 2016) can reduce the critical spacing, their effect on the interference is limited, and in a typical crowded display the critical spacing is often $0.3-0.5$ of eccentricity (Levi, 2008; Pelli et al., 2004).

A hallmark of crowding is inner-outer asymmetry; that is, in a radial display, an outer flanker (more peripheral) produces stronger interference than an inner one (closer to the fovea) (Banks Kenneth M Bachrach \& Larson, 1977; Bouma, 1970; Chaney, Fischer, \& Whitney, 2014; Dayan \& Solomon, 2010; Levi, 2008; Petrov \& Meleshkevich, 2011a, 2011b; Petrov, Popple, \& McKee, 2007; but see Strasburger \& Malania, 2013; Hans Strasburger, 2020).

Proposed models for explaining inner-outer asymmetry include, cortical magnification, receptive field size and spatial attention. According to the cortical magnification view, crowding is due to smaller critical distances in the periphery (Motter \& Simoni, 2007; Pelli, 2008). Thus, inner-outer asymmetry reflects smaller cortical distance between the outer flanker and the target compared to that between the inner flanker and the target. However, this view was challenged by the finding that inner-outer asymmetry is related to the reported item rather than the display. Namely, that within the same display (i.e., the same cortical distance), crowding interference is substantially reduced when observers report the outer item rather than the central one (Shechter \& Yashar, 2021). Moreover, the cortical magnification factor in $\mathrm{V} 1$ and other retinotopic areas is not large enough to explain the strong inner-outer asymmetry (Petrov et al., 2007). 
Receptive field (RF) size accounts of asymmetry rely on the fact that RF size increases at the visual periphery (Chaney et al., 2014; Dayan \& Solomon, 2010). For example, according to an optimal Bayesian model, the larger RF size of the outer flankers increases the number of RFs responding to the outer flanker compared to the inner one and thereby biases the Bayesian selection towards the outer flanker (Dayan \& Solomon, 2010).

According to the attentional selection view, attention is biased outward, leading to stronger interference of the outer flanker (Petrov \& Meleshkevich, 2011a). Support for this view comes from findings showing that asymmetry is reduced when attention is biased inward, either by a foveal task or by blocking stimulus eccentricity (Petrov \& Meleshkevich, 2011b). However, these manipulations involve other processes besides attention, such as task demand, stimulus uncertainty and difficulty. Moreover, in this study, as with most demonstrations of asymmetry, the target was flanked by a single flanker and such a crowded display may bias attention. So, it is still unclear whether and how the locus of covert spatial attention affects the inner-outer asymmetry in a typical crowded display where both flankers are presented simultaneously. In the present study we address this issue by manipulating covert attention.

Researchers manipulate transient spatial attention by presenting a peripheral cue that appears either at the target location (valid), a non-target location (invalid) or the fixation location (neutral). Attention's effect is assessed by comparing valid and neutral trials, and inattention's cost is measured by comparing neutral and invalid trials. Behavioral studies showed that attention enhances spatial resolution (e.g., (Yeshurun \& Carrasco, 1998)). Neurophysiological investigations suggest that attention shrinks cells' receptive field size over the attended location (reviewed by Anton-Erxleben \& Carrasco, 2013).

Despite the compelling evidence for attentional effect on spatial resolution, investigations of attentional manipulation in crowding yield mixed results. One study showed that attention decreases critical-spacing; i.e., the minimum spacing required for crowding (Yeshurun \& Rashal, 2010). Other studies, however, failed to demonstrate an effect on crowding (Scolari, Kohnen, Barton, \& Awh, 2007; Strasburger, 2005). One possible reason for this inconsistency is that locus of attention with respect to the target could have varied across these studies and therefore caused the attentional effect on crowding. Indeed, in Scolari et al., (2007), the cue appeared at the location of the target, while in (Yeshurun \& Rashal, 2010), the cue appeared closer to the fovea (inner flanker) than the target.

The exact locus of attention also has implications for investigating the predictions of the two competing asymmetry models. The attentional account predicts a direct positive effect 


\section{ATTENTION ASYMMETRY}

between attention and asymmetry. Namely, directing covert attention towards the outer flanker will increase asymmetry, whereas directing covert attention towards the inner flanker will decrease asymmetry. By contrast, the RF size view predicts a reduction of asymmetry when the locus of attention is at the outer-flanker location due to the shrinkage in RF size over the attended location.

Finally, the effect of locus of attention may vary across the different types of crowding errors. Investigations of the pattern of crowding errors revealed that crowding often leads to the misreporting of a flanker as the target (Ester, Klee, \& Awh, 2014; Freeman, Chakravarthi, \& Pelli, 2012; Harrison \& Bex, 2015; Strasburger \& Malania, 2013; Yashar, Wu, Chen, \& Carrasco, 2019). Crowding of two features, such as orientation and color, leads to misbinding errors; e.g., reporting an orientation of one item with the color of another (Yashar et al., 2019). Moreover, the effect of crowding on the perception of orientation, color, spatial frequency and motion is distinctive (Greenwood \& Parsons, 2020; Yashar et al., 2019). However, whether and how the locus of attention affects misreport and binding error is still unclear.

In the present study, we tested whether and how the locus of covert spatial attention affects crowding and its asymmetry. In two experiments observers estimated either the orientation of a grating (Gabor) stimulus (Experiment 1) or the tilt and color of a T-shaped object (Experiment 2) by reporting each feature in a continuous space. The target appeared on the horizontal meridian at $7^{\circ}$ eccentricity and was either alone (uncrowded) or along with two flankers, one on each side of the target on the horizontal meridian (a radial crowding). We used a center-to-center distance between the target and the flankers - that is, within the crowding window (I,e, $\mathrm{m}<0.5$ of eccentricity). To manipulate attention, we presented a precue (a circle) at one of four possible locations: fixation (neutral cue), the inner-flanker location (inner cue), the target location (target cue) and the outer-flanker location (outer cue). To assess the pattern of crowding errors, we compared the fitting of probabilistic mixture modeling to the error distributions. The results revealed that the effect of covert attention is contingent on the locus of attention within the crowded stimulus.

\section{Experiment 1}

\section{Method}

\section{Observers}

Eighteen students ( 6 males; age range $=22$ - 35 years, $M=26.66, S D=3.49$ ) from the University of Haifa participated in this experiment either for course credit or payment of 50 


\section{ATTENTION ASYMMETRY}

shekels (around $14 \$$ ) per hour. We estimated that a sample size of 16 observers is required to detect a medium to large effect with $80 \%$ power, given a .05 significance criterion based on a priori power analysis using effect sizes from previous studies (Shechter \& Yashar, 2021; Yashar et al., 2019). We collected data from two more observers because of possible dropouts or equipment failure. All observers were naïve to the research question and reported normal or corrected to normal vision, with no reported attention deficits. We obtained written informed consent from all observers before the experiment. The University Committee on Activities Involving Human Subjects at Haifa University approved all experimental procedures.

\section{Apparatus}

We used Matlab (The MathWorks, Inc., Natick, MA) with the Psychophysics Toolbox extensions (Kleiner et al., 2007) (Kleiner, Brainard, \& Pelli, 2007) to generate the stimuli and task. We ran the experiment on an iMac connected to a gamma-corrected 21 -in CRT monitor (with $1280 \times 960$ resolution and $85-\mathrm{Hz}$ refresh rate). We used Eyelink 1000 (SR Research), an infrared eye tracker to monitor and record eye movement and a SpectroCAL MKII (Cambridge Research Systems, UK) spectroradiometer to calibrate luminance and color. Observers were tested individually in a dimly lit room and used a mouse to generate responses. We used a chinrest to set the viewing distance of each observer at $57-\mathrm{cm}$.

\section{Stimuli and Procedure}

Figure 1 illustrates the sequence of events in a trial. All stimuli were presented on a gray background (luminance $53 \mathrm{~cd} / \mathrm{m}^{2}$ ). Each trial began with the fixation display, a black dot (subtending $0.24^{\circ}$ of visual angle, luminance $0.0073 \mathrm{~cd} / \mathrm{m}^{2}$ ) at the center of the screen. Following observer fixation for $300 \mathrm{~ms}$ a pre-cue (a black circle, $1.8^{\circ}$ in diameter and $0.5^{\circ}$ pen width) appeared for $50 \mathrm{~ms}$. The cue appeared at the location of the upcoming target (target cue $25 \%$ of the trials) or the less eccentric flanker (inner cue $25 \%$ of the trials) or the more eccentric flanker (outer cue $25 \%$ of the trials). In the remaining $25 \%$ of the trials, the cue appeared at fixation (neutral cue). After an inter stimulus interval (ISI) of 50ms, a peripheral target appeared for $100 \mathrm{~ms}$. The target was a Gabor: a sinusoidal grating (1.5 cycles per degree) with a Gaussian envelope (SD of $0.65^{\circ}$ ) and $75 \%$ contrast (the size was about $1.8^{\circ}$ in diameter). The target was located on the horizontal meridian with $7^{\circ}$ of eccentricity either in the left or in the right hemifield. The target appeared either alone (uncrowded-display condition) or flanked by two Gabors (crowded-display condition). The flankers appeared one on each side of the target on the horizontal meridian. The center-to-center spacing between the target and the flankers was $2.3^{\circ}$. 


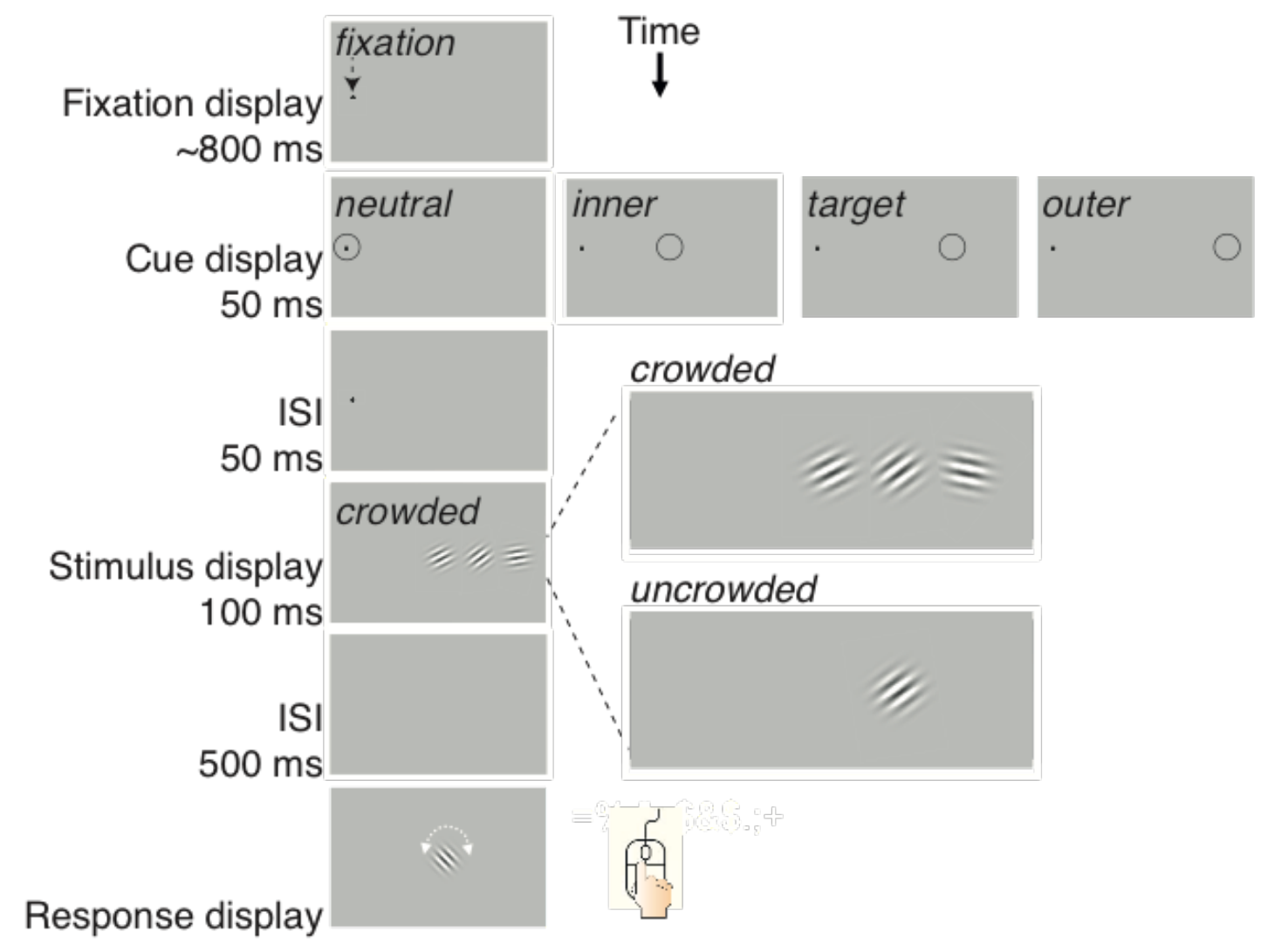

Figure 1. Illustration of the sequence of events within a trial in Experiment 1. Here, only the right hemifield of the display is shown; i.e., the fixation mark was at the center of the screen. The cue appeared either at fixation (neutral), the inner-flanker location, the target location or the outerflanker location. The target ( $7^{\circ}$ eccentricity) appeared alone (uncrowded) or radially flanked by two Gabors $\left(2.5^{\circ}\right.$ center to center distance). Observers estimated the target orientation by adjusting the probe using a mouse.

Target and flanker orientation were randomly sampled from a circular parameter space of 180 values evenly distributed between $1^{\circ}$ and $180^{\circ}$, with the restriction that, in each trial, Gabors' orientation differed by at least $15^{\circ}$ from one another. A blank interval of $500 \mathrm{~ms}$ followed the stimulus display, then the response display appeared and remained on the screen until the observers completed their response. The response display comprised a probe (a Gabor at the center of the screen). Observers were asked to estimate the target orientation by adjusting the orientation of the probe using the mouse. Each condition had 100 trials (800 trials overall). Each observer completed ten blocks of 80 trials in one session. 


\section{ATTENTION ASYMMETRY}

In each block, there were 20 trials from each of the four cue conditions. The experiment began with a 40-trial practice block. Observers were encouraged to take a short break between blocks. To monitor eye fixation and stimulus eccentricity, we used online eyetracking. We terminated trials in which the observer broke fixation and reran them at the end of the last block ( $>2^{\circ}$ from fixation).

\section{Models and analysis}

We calculated the estimation error in each trial by subtracting the target's true value from the estimated value. First, for each observer in each condition, we assessed report bias and report precision by calculating the mean and the inverse of the standard deviation (std) in radians of the error distribution, respectively.

We then analyzed the error distributions by individually fitting probabilistic-mixture models developed from the standard and standard-with-misreporting models (Bays, Catalao, \& Husain, 2009; Zhang \& Luck, 2008).

For uncrowded-display trials we fitted the standard model (Equation 1), which uses a von Mises (circular) distribution to describe the probability density of the pooling estimation of the target's orientation and a uniform component to reflect the guessing in estimation. The model has two free parameters $(\gamma, \sigma)$, In this model, the probability of reporting a feature value $p(\hat{\theta})$ is

$$
p(\hat{\theta})=(1-\gamma) \phi_{\sigma}(\hat{\theta}-\theta)+\gamma\left(\frac{1}{180}\right)
$$

Where $\hat{\theta}$ is the value of the reported feature and $\theta$ is the actual value of the target feature, $\gamma$ is the proportion of trials in which observers are randomly guessing (guessing rate), $\phi_{\sigma}$ is the von Mises distribution with a standard deviation $\sigma$ (variability) and a mean of 0 .

For crowded-display trials, we compared the fitting of models that included a component of misreporting a flanker as the target.

The one-misreport model (Equation 2) has three free parameters $(\gamma, \sigma, \beta)$. The model adds a misreporting component to the standard model. The misreport component describes the probability of reporting one of the flankers to be the target. In this model, the probability of reporting a feature value is 


$$
p(\hat{\theta})=(1-\gamma-\beta) \phi_{\sigma}(\hat{\theta}-\theta)+\gamma\left(\frac{1}{180}\right)+\frac{1}{m} \beta \sum_{i=1}^{m} \phi_{\sigma}\left(\hat{\theta}-\varphi_{i}\right)
$$

where $\beta$ is the probability of misreporting a flanker as the target, and $\varphi_{i}$ is the actual value of the $i$ flanker and $m$ is the total number of flankers. The variability of the distribution around each stimulus was assumed to be the same.

The two-misreport model (Equation 2) has four free parameters $\left(\gamma, \sigma, \beta^{\text {In }}, \beta^{\text {Out }}\right)$. The model adds two misreporting components to the standard model. Each misreport component describes the probability of reporting one of the flankers to be the target. In this model, the probability of reporting a feature value is

$$
p(\hat{\theta})=\left(1-\gamma-\beta^{I n}-\beta^{\text {Out }}\right) \phi_{\sigma}(\hat{\theta}-\theta)+\gamma\left(\frac{1}{180}\right)+\beta^{I n} \phi_{\sigma}\left(\hat{\theta}-\varphi^{I n}\right)+\beta^{\text {Out }} \phi_{\sigma}\left(\hat{\theta}-\varphi^{\text {Out }}\right)
$$

Where $\beta^{I n}$ is the probability of misreporting the inner flanker as the target, and $\beta^{\text {Out }}$ is the probability of misreporting the outer flanker as the target, $\varphi^{I n}$ and $\varphi^{\text {Out }}$ are the actual value of the inner flanker and the outer flanker, respectively.

We used the MemToolbox (Suchow, Brady, Fougnie, \& Alvarez, 2013) tor model fitting and comparison. To compare models, we calculated Akaike information criterion with correction (AICc) for the individual fits. We calculated the target reporting rate as $P t=(1-\gamma)$, $P t=(1-\gamma-\beta)$ and $P t=\left(1-\gamma-\beta^{I n}-\beta^{\text {Out }}\right)$ in the standard model, one-misreport model and two-misreport model, respectively.

We performed a 2X4 analysis of variance (ANOVA) with display condition (uncrowded display vs. crowded display) and cue condition (neutral, inner, valid, outer) as within-subject factors on precision and parameters of the best performing model. To test our main hypothesis, we performed a four-way ANOVA with cue position as within-subject factors on the best fitting model parameters. 


\section{Results and discussion}

Figure 2A plots the distribution of errors for the uncrowded- and crowded-display trials. Mean report bias in all conditions was within the range of $\pm 2^{\circ}$, indicating that there was no systematic report bias (Supplementary information [SI], Table S1).

As expected, report precision was higher in uncrowded-display trials than in crowded-display trials (Figure 2B). There were no other significant effects on precision, a ps>.72 (SI, Table S1).

\section{Model fitting results}

For crowded-display trials, as indicated by the mean AICc (SI, Table S2), the two-misreport model outperformed the standard model and the one-misreport models, suggesting that misreport rate differed between the inner and the outer flankers (Figure 3A). Next, we analyzed model parameters (Table S3, Figure 2D-E and Figure 3) of the standard model in uncrowded-display trials and the two-misreport model in crowded-display trials.

Crowded display increased variability $(\sigma), F(1,15)=14.99, p=0.002, \eta^{2} p=0.5$ (Figure 2C), and guesses $(\gamma), F(1,15)=6.97, p=0.019, \eta^{2} p=0.32$, and decreased target reports $(P t)$, $F(1,15)=113.93, p<0.001, \eta^{2} p=0.88$ (Figure 2E). See Supplementary analysis for the remaining 2X4 ANOVAs results on all parameters.
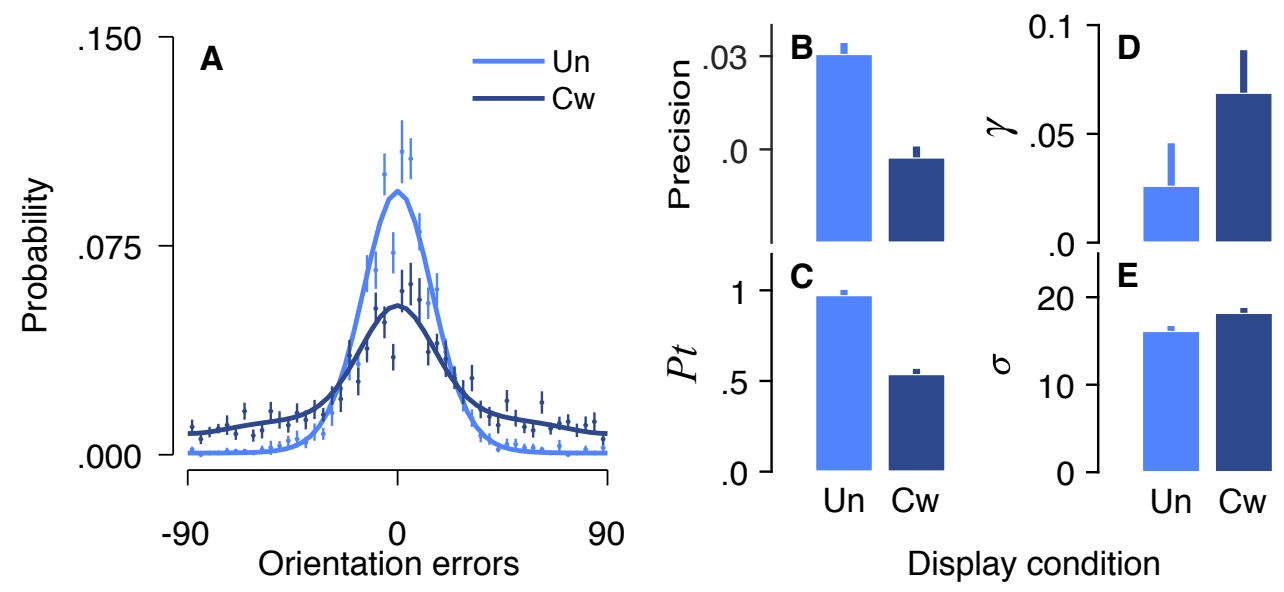

Figure 2. Uncrowded- vs. crowded-display trials in Experiment 1. (A) We plotted mean error distribution in the uncrowded-display trials and the crowded-display trials (solid lines are the fitted models). (B) We calculated precision as the inverse of the SD of the data. (C - E) We plotted the means fitted parameters of the individual fits of the two-misreports model as a function of display condition. Un=uncrowded-display trials, $\mathrm{Cw}=$ crowdeddisplay trials. Error bars \pm 1 within subject $\mathrm{SE}$. 
Next, we analyzed the cuing effect in crowded-display trials. Planned four way ANOVA in crowded-display trials showed a higher $P t$ in inner cue trials than in neutral trials, $t(15)=2.64$, $\mathrm{p}<0.018$, and lower $P t$ in outer trials than in inner trials, $\mathrm{t}(15)=2.17, \mathrm{p}=0.046$. Target report rate in target-cue trials was not significantly different that in neutral trials, $p>0.5$. Thus, in a crowded display, pre-cueing the crowding stimulus either increased or decreased target report rates depending on whether we pre-cued the inner or the outer flanker. Cue position modulated misreport of the outer flanker $\left(\beta^{\text {Out }}\right), \mathrm{F}(3,45)=8.42, \mathrm{p}<0.001$, but not the inner flanker $\left(\beta^{I n}\right), \mathrm{F}<1$ (Figure 3C). Planned comparisons revealed lower $\beta^{\text {Out }}$ in inner cue trials compared to neutral trials, $\mathrm{t}(16)=2.64, \mathrm{p}=0.018$, and higher $\beta^{\text {Out }}$ in outer cue trials compared to neutral trials, $\mathrm{t}(13)=2.17, \mathrm{p}=0.046$. $\beta^{\text {Out }}$ did not significantly differ between target cue and neutral cue, $t<1$. These findings show that the chance of reporting the outer flanker increased with the proximity of the outer flanker to the locus of focal attention, demonstrating a positive relation between attention and inner-outer asymmetry.

neutral cue

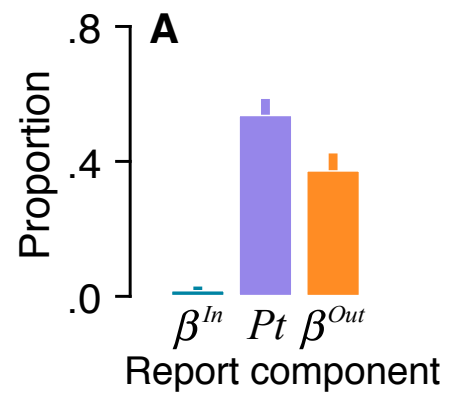

peripheral cue - neutral cue
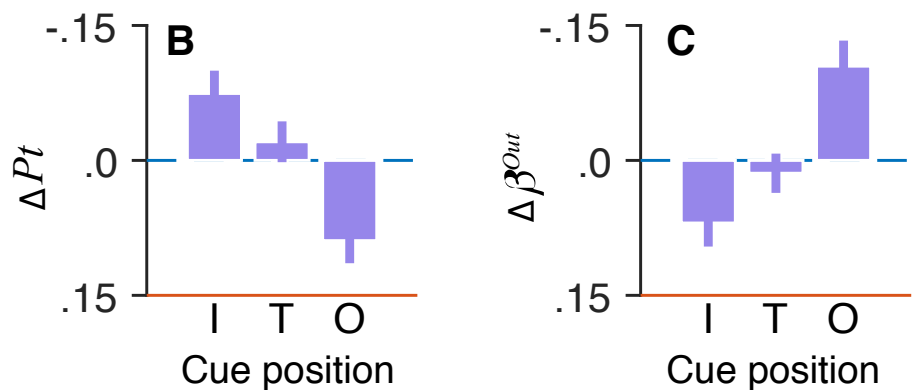

Figure 3. Misreport errors and cuing effect in crowded-display trials of Experiment 1. (A) We plotted mean report rate for the inner flanker, the target and the outer flanker in the neutral cue condition. We plotted mean cueing effect on (B) target report rate and (C) misreports of the outer flankers. We calculated the cueing effect by subtracting the neutral cue from each peripheral cue condition: $\mathrm{I}=\mathrm{inner}, \mathrm{T}=$ target and $\mathrm{O}=$ outer. Error bars \pm 1 within subject SE. 


\section{Experiment 2}

In Experiment 2, we extended our investigation to the process of feature binding. A recent study (Yashar et al., 2019) tested the effect of crowding on feature binding; i.e., the integration of feature dimensions (e.g., tilt, color and SF) to a coherent object. Observers performed a double report task by estimating both the tilt (fully circular space $0^{\circ}-360^{\circ}$ ) and the color (on a color wheel $0^{\circ}-360^{\circ}$ ) of a T-shaped target. In a crowded display, observers misreported tilt or colors in an independent manner. That is, observers often reported presented features accurately, but in an inaccurate conjunction. These findings suggest that crowding disrupts the integration of features into a coherent object, leading to what is known as mis-binding or 'illusory conjunction' (Treisman \& Schmidt, 1982).

Classical visual attention views argue that spatial attention plays a critical role in feature binding. Indeed, when covert attention was disrupted, mis-binding errors were reported in uncrowded displays (i.e., object spacing was outside the crowding window) (A. M. Treisman \& Gelade, 1980; A. Treisman \& Schmidt, 1982). In Experiment 2 we tested the effect of covert spatial attention on both crowding and binding errors. If binding errors in crowding are due to diffused attention, then directing spatial attention should modulate binding errors.

\section{Method}

The method was the same as in Experiment 1 except for the following.

\section{Observers}

Nineteen students ( 7 males: age range 23 - 37 years, $M=28.37, S D=4.34$ ).

\section{Stimuli and Procedure}

Figure 4 illustrates the sequence of events in a trial. The pre-cue appeared at one of three possible locations: fixation (neutral), inner flanker or outer flanker. The target and the flankers were T-shaped items, each subtending $1.8^{\circ} \times 1.8^{\circ}$ and drawn with a $0.3^{\circ}$ stroke. The tilt and color of the target and the flankers were each independently selected at random from two circular parameter spaces. Target and flanker tilt were randomly selected out of 360 values evenly distributed between $1^{\circ}$ and $360^{\circ}$. The color was randomly selected out of 360 values evenly distributed along a circle in the DKL color space (Derrington, Krauskopft, Lenniet, Kra, \& Lennie, 1984). We followed the same color space calibration as Yashar et al., (2019) (see Yashar et al., 2019 Supplementary Information). Stimuli color and background were equiluminant. 


\section{ATTENTION ASYMMETRY}

The color response displays included a color wheel $\left(2^{\circ}\right.$ thick with an inner radius of $\left.5^{\circ}\right)$ containing 360 colors. Observers were asked to estimate the target color by selecting a color on the color wheel using the mouse curser. During response, a visual presentation of the selected color was presented at the center of the screen. As in Experiment 1, observers estimated the target tile by rotating a T-shaped item at the center the screen using the mouse.

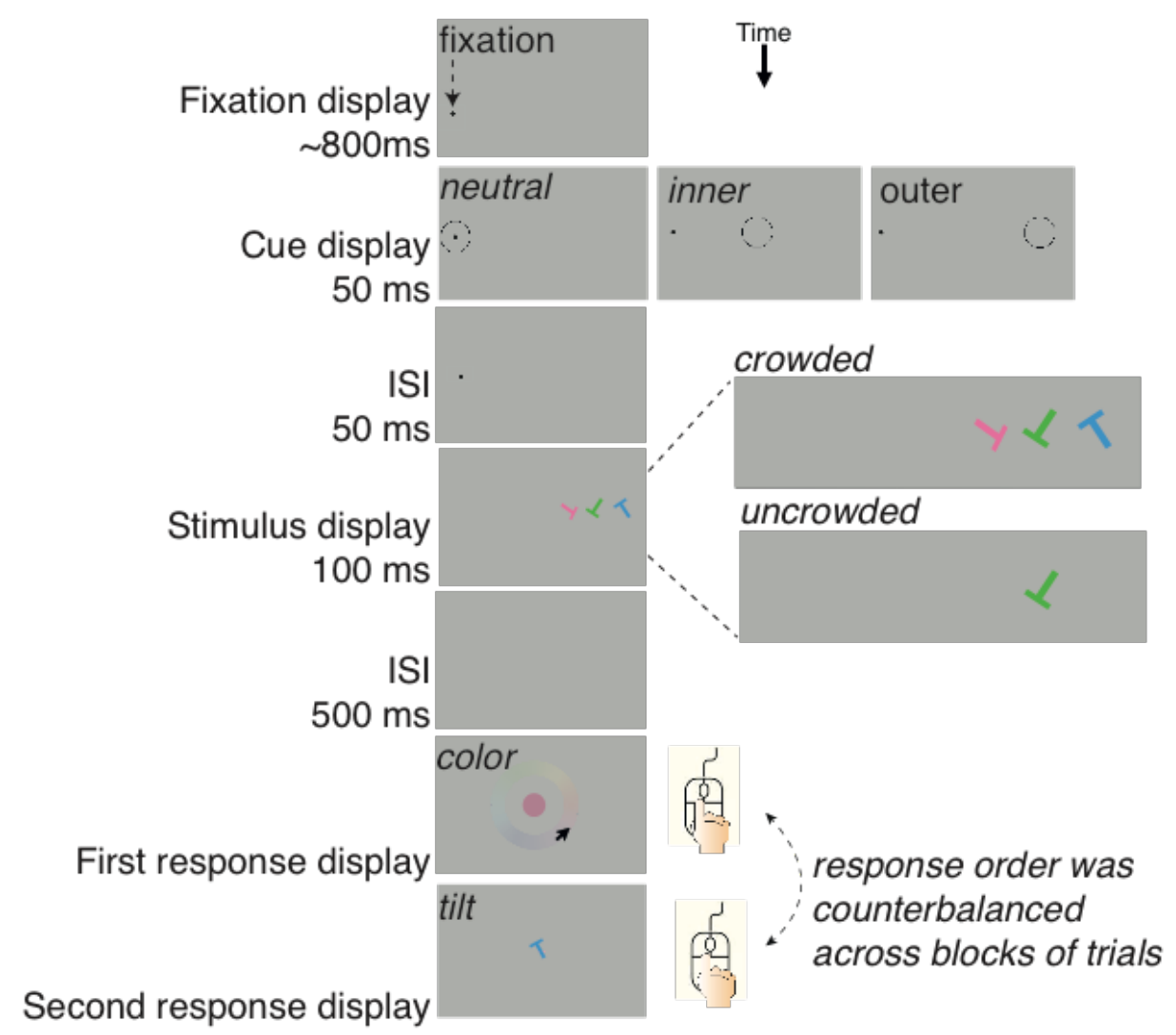

Figure 4. Illustration of the sequence of events within a trial in Experiment 2. Fixation point, here on the left, was presented at the center of the screen. The cue appeared at fixation (neutral), at the inner-flanker location or at the outer-flanker location. Observers estimated the target color and orientation by adjusting the probe using a mouse.

In both response types, a final report was made by clicking the mouse. The response order (tilt and color) alternated every block of 150 trials. In each of the three cue positions there were 200 crowded-display trials and 100 uncrowded-display trials (900 trials in total). We encouraged observers to take a short break every 50 trials. The experiment began with a 40-trial practice block. 


\section{Models and analysis}

To analyze each feature space separately, we performed the same model fitting procedure as described in Experiment 1. In addition to the standard model, the one-misreport model and the two-misreport model, we also fitted these models with a target bias component: the bias standard model, the bias one-misreport model and the bias two-misreport model. These models were similar to the regular models except that the mean $(\mu)$ of the von Mises distribution around the target $\phi_{\sigma, \mu}$ was a free parameter.

Joint-distribution models. To analyze binding errors, we fitted a joint-standard mixture model (Bays, Wu, \& Husain, 2011; Dowd \& Golomb, 2019) to the joint distribution of tilt and color. For uncrowded-display trials, each feature dimension report could come from uniform or target distributions, leading to four possible report combinations of tilt and color distribution (Table 1, rows 1-4, joint-standard model). For crowded-display trials, each feature dimension report could come from either one of four distributions: a uniform, a Gaussian over the target, a Gaussian over the inner flanker or a Gaussian over the outer flanker. Because we had two feature dimensions, the total number of possible distribution combinations of tilt and color was 16 (Table 1, rows 1-16, joint-misreport model). Each joint model also included a von Mises variability component for each feature dimension $\left(\sigma_{t}, \sigma_{c}\right)$. Overall, the joint-standard model has five free parameters, and the joint-misreport model had 17 free parameters.

We used the MCMC function in the MemFit toolbox to individually fit the models in each crowding and cue condition. To simplify the analysis of the joint-misreport model (16 components) in crowded-display trials, we grouped components into four categories of reports: (1) Bound target (Table 1, row 1), which related to the report of both tilt and color of the target (2) Feature error (Table 1, rows 2-8), which related to any guessing component, (3) Binding errors (Table 1, rows 9-14), which related to misreport of different items (e.g., the target tilt with a flanker color); (4) Object error (Table 1, rows 15-16), which related to misreporting both features of the same flanker. Note that both object error and target report reflect correct binding. To test for the effect of covert spatial attention on binding, we analyzed the effect of cue position on each of the four error types. We performed a threeway, repeated measure ANOVA with cue position as a within-subject factor on each component category. To test for the effect of cue position on correct binding in uncrowded display trials, we performed a three-way repeated measure ANOVA with cue position as within subject factor on bound target rate (Table 1, row 1) of the standard joint model. 
Table 1. The mixture components of the joint-standard model (rows 1-4) and the joint-misreport model (rows 1-16).

\begin{tabular}{|c|c|c|c|c|}
\hline Component & & & Mixture & Probability Density \\
\hline Category & Tilt & Color & Component & Function \\
\hline
\end{tabular}

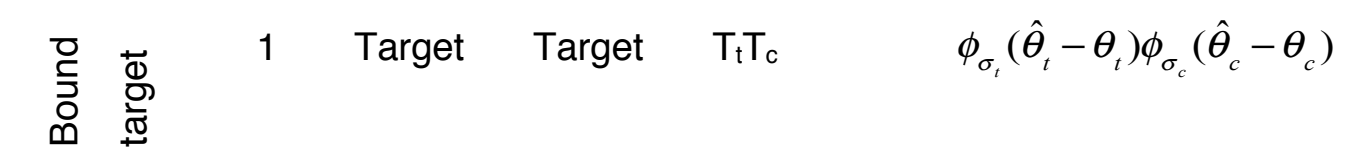

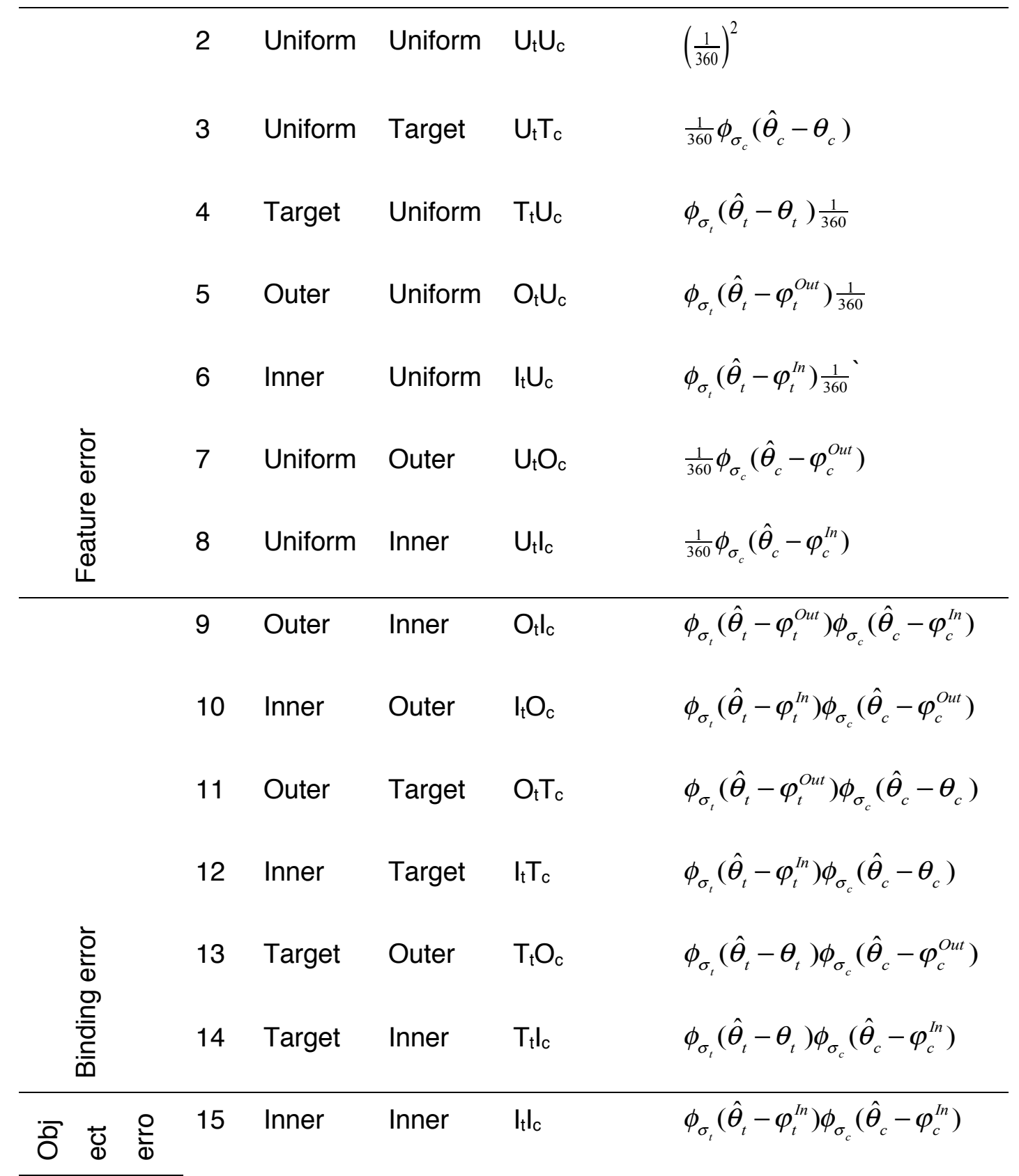


16 Outer Outer $\quad \mathrm{O}_{\mathrm{t}} \mathrm{O}_{c} \quad \phi_{\sigma_{t}}\left(\hat{\theta}_{t}-\varphi_{t}^{\text {Out }}\right) \phi_{\sigma_{c}}\left(\hat{\theta}_{c}-\varphi_{c}^{\text {Out }}\right)$

\section{Results and discussion}

Figure 4A plots the distribution of errors for the uncrowded- and crowded-display trials for tilt and color. For either tilt or color reports, precision was higher in uncrowded-display trials than in crowded-display trials, $F(1,18)=78.75, p<0.001, \eta^{2} p=0.81, F(1,18)=24.98, p<$ $0.001, \eta_{p}^{2}=0.58$, respectively. The main effect of cue position on precision was significant for color, $F(2,36)=5.15, p<0.019, \eta^{2}{ }_{p}=0.22$. No other effect was significant on precision, and no effect was significant on report bias, al ps $>0.1$ (SI, Table S4).

\section{Model fitting results}

We removed one observer from the model fitting analysis due to a high guessing rate (>.50).

For each feature dimension, we analyzed the fitted parameters of the bias-mixture model and the bias two-misreport models in uncrowded- and crowded-display trials, respectively (see models AICcs and all fitted parameters values in Supplementary Table S5-7).
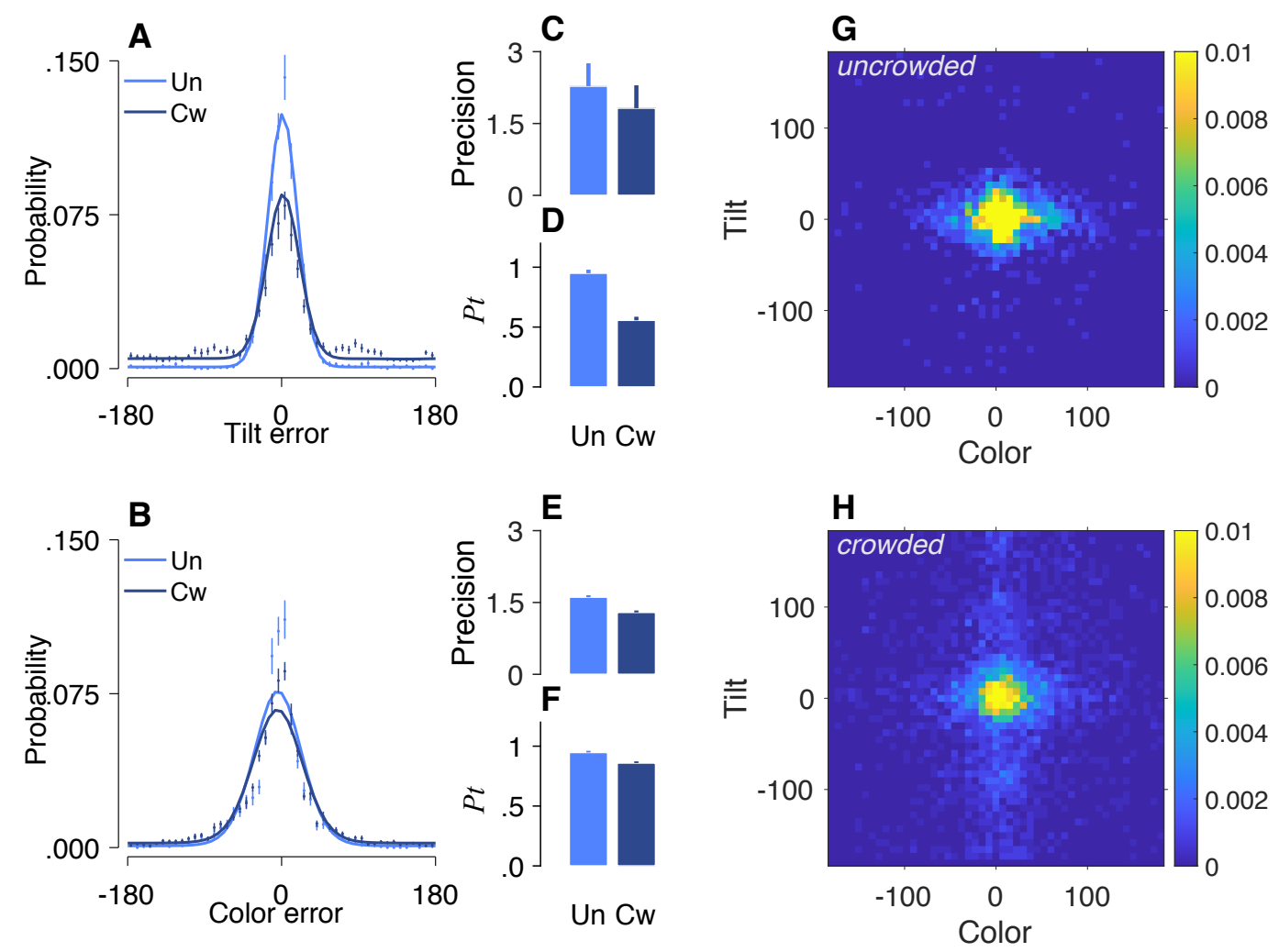

Figure 5. Uncrowded-display trials vs. crowded-display trials in Experiment 2. (A \& B). We plotted mean error distributions (dots) and model 


\section{ATTENTION ASYMMETRY}

fits (solid lines) for (A) tilt and (B) color reports. (C - F) We plotted mean precision in uncrowded- and crowded-display trials for (C) tilt and $€$ color, and mean target report rates in uncrowded- and crowded-display trials for (D) tilt and $(\mathbf{F})$ color. $(\mathbf{G} \& \mathbf{H})$ We plotted the joint distribution of tilt and color for $(\mathbf{G})$ uncrowded-display trials and $(\mathbf{H})$ crowded-display trials. Error bars are \pm 1 within subject SEM.

Tilt

Crowding increased tilt $\sigma$ (Figure 5), $F(1,17)=4.58, p=0.047, \eta^{2}{ }_{p}=0.21$. All other effects on tilt $\sigma$ were not significant, $\mathrm{p}>0.1$. Pt were lower in crowded-display trials than in uncrowded-display trials (Table S6), $F(1,17)=87.39, p<0.001, \eta^{2}{ }_{p}=0.83$.

Next, we analyzed the cueing effect in crowded-display trials. Figure 6A plots $\beta^{I n}, P t$ and $\beta^{\text {out }}$ in neutral cue trials in crowded-display trials. Cue position significantly affected $\beta^{\text {out }}$, $F(2,34)=9.07, p<0.001$. Planned comparisons revealed lower $\beta^{\text {out }}$ in inner-cue trials than in neutral cue, $t(17)=2.21, p=0.041$, Chens' $D=0.52$, and higher $\beta^{\text {out }}$ in outer-cue trials than in neutral trials, $t(17)=2.27, p<0.035$, Cohens' $\mathrm{D}=0.53$ (Figure 6C). The effect of cue position on $P t$ (Figure 6B) and $\beta^{I n}$ was not significant, $F(2,34)=2.93, p=0.065$, and $F(2,34)=2.94, p=0.066$, respectively.
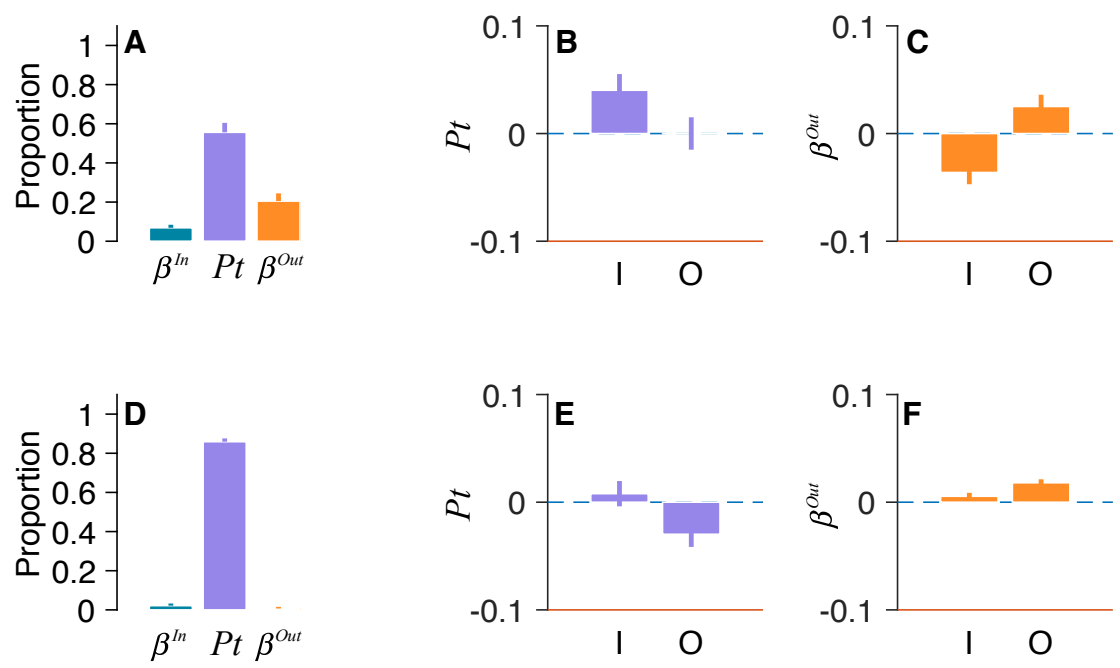

Report component
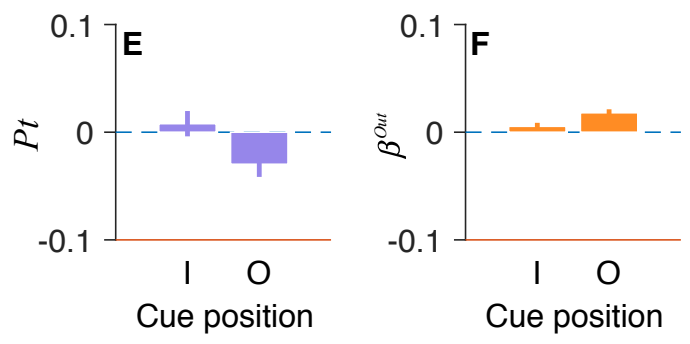

Figure 6. Misreport errors of the bias two-misreport model for tilt (A-C) and color (D-F) in Experiment 2. (A \& D) We plotted mean report for the 
inner flanker $\left(\beta^{I n}\right)$, the target $(P t)$ and the outer flanker $\left(\beta^{O u t}\right)$ in the neutral cue condition. We plotted mean cueing effect on $(B \& E)$ target report rates and ( $C \& F)$ misreports of the outer flankers. We calculated the cueing effect by subtracting the neutral cue from each peripheral cue condition: $I=i n n e r, T=$ target and $\mathrm{O}=$ outer. Error bars \pm 1 within subject SE.

Color

Crowding increased color $\sigma$ and reduced $P t, F(1,17)=5.27, p=0.034, \eta^{2} p=0.23$ (Figure $5 E$ ), and $F(1,17)=7.18, p=0.015, \eta^{2} p=0.30$ (Figure $5 F$ ), respectively. No other main effect or interaction was significant, al ps $>0.1$.

In crowded-display trials, there was no main effect of cue position on color $\beta^{I n}, \mathrm{~F}<1$. As with tilt, cue position significantly affected $\beta^{\text {Out }}, \mathrm{F}(2,34)=4.79, \mathrm{p}=0.014, \eta^{2} \mathrm{p}=0.22$.

Planned comparisons revealed that $\beta^{\text {Out }}$ was higher in outer-cue trials than in inner-cue trials, $\mathrm{t}(17)=2.71, \mathrm{p}=0.014$, and higher in outer-cue trials than in neutral-cue trials, $\mathrm{t}(17)=$ 2.75, $\mathrm{p}=0.013$. For target report rate, a planned comparison revealed higher $P t$ in innercue trials than in outer-cue trials, $t(17)=2.26, p=0.036$. These findings show that spatial attention directly modulates the color misreport rate of the outer flanker.

\section{Joint-distribution models}

Next, we tested the effect of cue position on binding error by analyzing the fitted parameters of the joint-distribution models (Table S8). For the joint-standard model in uncrowdeddisplay trials, there was no significant effect of cue position on bound target report rate: $F<1$. Figures 5G \& 5H depict the joint distribution of tilt and color for uncrowded- and crowdeddisplay trials, respectively.

A

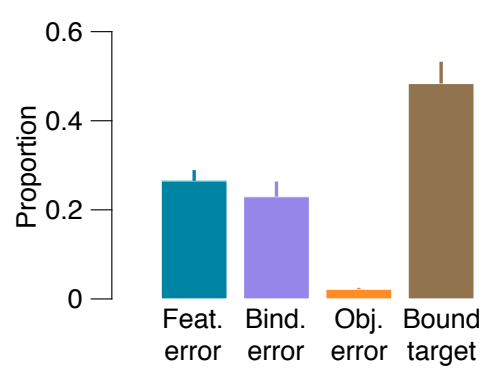

B

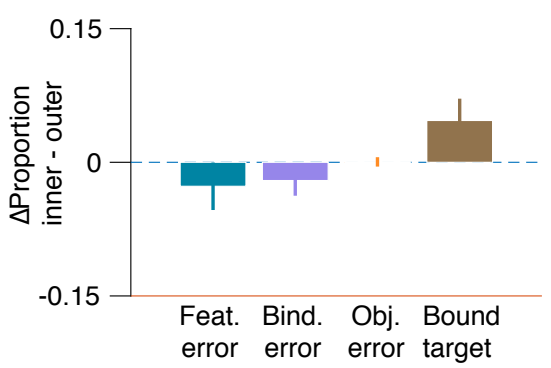

Figure 7. Joint feature report rates in Experiment 2. (A) Mean report rates for each report-component category of the joint-misreport model. (B) For each component, we plot the cueing effect by subtracting report 
proportion in the outer-cue from the inner-cue trials. Feat. error $=$ feature error, Bind. error $=$ binding error, Obj. error $=$ object error, and Bound target $=$ reporting the target in both features. Error bars are \pm 1 within subject SE.

Next, we analyzed the cuing effect on the join-misreport-model components in crowdeddisplay trials. Figure 7A shows the mean rate for each mixture component category in neutral trials. Bound target report rate was higher in the inner-cue position compared to both the neutral-cue position, $t(17)=2.29, p=0.035$, and the outer-cue position, $t(17)=2.21, p=0.04$ (Figure 7B). These findings reflect the overall increase in target report rate in inner-cue trials within each feature space. Interestingly, when cue position was tested on each of the three error types, cue position did not significantly affect the feature errors, binding errors or object errors, all ps $>0.18$ (Figure 7B).

\section{General discussion}

The results show that the locus of covert attention within the crowded stimulus (i.e., inner, target or outer locations) determines target identification. First, as in the Shechter and Yashar (2021) study, instead of the target, observers often misreported the orientation or tilt of the outer flanker rather than the inner one, which demonstrates inner-outer asymmetry in a typical display of radial crowding. Second, compared to maintaining allocation at fixation, attending to the target location did not affect target performance. Interestingly, attending to the inner-flanker location - a more foveal location than the target - increased the target identification and reduced the asymmetry. Finally, attending to the outer flanker reduced the target identification and increased the asymmetry.

This direct and positive relationship between covert attention and inner-outer asymmetry is consistent with the attentional bias account (Petrov \& Meleshkevich, 2011b) and inconsistent with the receptive field size view (Chaney et al., 2014; Dayan \& Solomon, 2010). Whereas attentional accounts predict an increase in asymmetry when the locus of attention is the outer-flanker location, the receptive field size view predicts a decrease in asymmetry in outer-cue trials due to the reduction in receptive field size over the outer flanker.

\section{Spatial attention and crowding}

The present findings explain the inconsistent results of spatial attention investigations in crowding and attribute them to variations in the locus of attention. Consistent with the present study, previous studies showed that cueing attention at the target location did not reduce crowding interference (Scolari et al., 2007; Strasburger \& Malania, 2013), whereas 


\section{ATTENTION ASYMMETRY}

cueing attention at location inner to the target reduced crowding interference (Yeshurun \& Rashal, 2010).

Investigations of the spatial attention effect on basic signal processing typically display a simple stimulus around threshold levels, often by reducing stimulus strength (reviewed by (Carrasco, 2011). Here, to test the attentional effect on crowding alone, we used a high contrast super-threshold target. Thus, we did not expect to find an attentional effect in uncrowded-display trials in which performance was relatively at ceiling levels (i.e., Pt close to 1$)$.

\section{Crowding, binding and attention}

The present study has implications for the feature-binding issue. First, this study replicates Yashar's et al. (2019) results by showing that crowding errors reflect binding errors. Specifically, when observers had to report the tilt and color of a T-shaped target, they often misreported the tilt of one item with the color of another item. Here, a joint-misreport mixture model revealed that observers performed binding errors (i.e., reporting one feature from one object and the second feature from another object) or feature errors (i.e., reporting one or two feature values unrelated to a presented object - a guess). Notably, only a small percentage of trials reflected object error (i.e., reporting two features of the same flanker), suggesting that misreport errors reflect feature integration errors rather than confusion between coherent objects. This finding suggests that crowding is due to excessive integration processes and supports pooling models (Freeman et al., 2012; Freeman \& Simoncelli, 2011; Keshvari \& Rosenholtz, 2016; Rosenholtz, Yu, \& Keshvari, 2019).

Second, the results provide insight into the role of spatial attention in feature binding. A prominent view of feature-binding and attention is the feature integration theory (A. M. Treisman \& Gelade, 1980), according to which attention operates as the 'glue' that binds features together. Thus, this view predicts that allocating covert attention towards the crowded stimulus will reduce binding errors-namely, attention would lead to higher reports of a coherent target (bound target) and coherent flankers (object errors).

However, the present study results are inconsistent with this prediction. First, they show that binding errors in a crowded display are persistent even when covert attention is allocated toward the crowded stimulus. Second, they show that cueing covert attention to the crowded stimuli affected feature and binding errors, but not object errors. In other words, when observers misreported a flanker feature as the target, covert spatial attention did not 'glue' 


\section{ATTENTION ASYMMETRY}

the other feature dimension to generate a coherent object perception and reports of the other feature of the same flanker (object errors).

This finding is inconsistent with previous studies that tested the effect of spatial attention on binding errors and showed that cueing attention decreased binding errors in a conjunction detection task (Briand, 1998; Prinzmetal, Presti, \& Posner, 1986). However, these studies did not monitor eye movement and used a cue-stimulus stimulus onset asynchrony (SOA) of 227-250 ms, which was enough time for a saccade (e.g., (Mayfrank, Kimmig, \& Fischer, 1987)). Thus, it is unclear whether the cueing effect is due to overt-rather than covertattention. Here, by monitoring eye movement using an eye tracker, we were able to test the effect of covert attention per se on feature binding. We showed that the effect of covert attention on feature binding is limited to the task relevant item - i.e., the target.

Moreover, we showed both the cost and benefit of attentional allocation in a crowded display by testing various cue positions. Therefore, our feature binding findings go beyond a particular cue-target special relation. In Experiment 2, we used two cue positions, inner cue and outer cue, which we selected to maximize attentional cost and benefit based on Experiment 1. The pattern of cue position effect on crowding errors was consistent across Experiments. Thus, it is unlikely that adding target-cue position in Experiment 2 would have changed the pattern of feature binding results.

Note that our findings mainly apply to bottom-up covert attention. However, top-down attention may still play a critical role in feature-binding. Indeed, according to a prominent view, crowding is due to reduced attention resolution in the periphery (Chakravarthi \& Cavanagh, 2007; He, Cavanagh, \& Intriligator, 1996; Intriligator \& Cavanagh, 2001; Tripathy $\&$ Cavanagh, 2002). This view assumes that the minimum size of the attentional selection region is larger in the periphery. Thus, when two or more items fall into the selection region, they are indistinguishable. Given that the selection region reflects top-down attention that differs from the bottom-up attention manipulated by the cue, our findings do not challenge the attentional selection view of crowding. Thus, reduced top-down attentional resolution may be responsible for binding errors in crowding.

\section{Conclusion}

The present study results reveal the important role of covert spatial attention in inner-outer asymmetry. The findings are consistent with the attentional bias account of inner-outer asymmetry and inconsistent with the receptive field size account. Our study also 


\section{ATTENTION ASYMMETRY}

demonstrates a strong link between crowding and feature binding/integration and shows that crowding errors reflect binding errors. 


\section{References}

Anton-Erxleben, K., \& Carrasco, M. (2013). Attentional enhancement of spatial resolution: Linking behavioural and neurophysiological evidence. Nature Reviews Neuroscience, Vol. 14. https://doi.org/10.1038/nrn3443

Banks, W. P., Bachrach, K. M., \& Larson, D. W. (1977). The asymmetry of lateral interference in visual letter identification. Perception \& Psychophysics, 22(3), 232-240. https://doi.org/10.3758/BF03199684

Bays, P. M., Catalao, R. F. G., \& Husain, M. (2009). The precision of visual working memory is set by allocation of a shared resource. Journal of Vision, 9(10). https://doi.org/10.1167/9.10.7

Bays, P. M., Wu, E. Y., \& Husain, M. (2011). Storage and binding of object features in visual working memory. Neuropsychologia, 49(6), 1622-1631.

https://doi.org/10.1016/j.neuropsychologia.2010.12.023

Bouma, H. (1970). Interaction Effects in Parafoveal Letter Recognition. Nature, 226(5241), 177-178.

Briand, K. A. (1998). Feature integration and spatial attention: More evidence of a dissociation between endogenous and exogenous orienting. Journal of Experimental Psychology: Human Perception and Performance, 24(4), 12431256. https://doi.org/10.1037/0096-1523.24.4.1243

Carrasco, M. (2011, July 1). Visual attention: The past 25 years. Vision Research, Vol. 51, pp. 1484-1525. https://doi.org/10.1016/j.visres.2011.04.012

Chakravarthi, R., \& Cavanagh, P. (2007). Temporal properties of the polarity advantage effect in crowding. Journal of Vision, 7(2), 11.1-13. https://doi.org/10.1167/7.2.11

Chaney, W., Fischer, J., \& Whitney, D. (2014). The hierarchical sparse selection model of visual crowding. Frontiers in Integrative Neuroscience, 8(SEP), 73. https://doi.org/10.3389/fnint.2014.00073

Chung, S. T. L. (2007). Learning to identify crowded letters: does it improve reading speed? Vision Research, 47(25), 3150-3159.

https://doi.org/10.1016/j.visres.2007.08.017

Dayan, P., \& Solomon, J. A. (2010). Selective Bayes: Attentional load and crowding. Vision Research, 50(22), 2248-2260. https://doi.org/10.1016/j.visres.2010.04.014

Derrington, A. M., Krauskopft, J., Lenniet, P., Kra, J., \& Lennie, P. (1984). 241 With 15 text-figure8 CHROMATIC MECHANISMS IN LATERAL GENICULATE NUCLEUS OF MACAQUE. In J. Physiol (Vol. 357).

Dowd, E. W., \& Golomb, J. D. (2019). Object-Feature Binding Survives Dynamic Shifts of Spatial Attention. Psychological Science, 30(3), 343-361.

Ester, E. F., Klee, D., \& Awh, E. (2014). Visual crowding cannot be wholly explained 
by feature pooling. Journal of Experimental Psychology: Human Perception and Performance, 40(3), 1022-1033. https://doi.org/10.1037/a0035377

Freeman, J., Chakravarthi, R., \& Pelli, D. G. (2012). Substitution and pooling in crowding. Attention, Perception, and Psychophysics, 74(2), 379-396. https://doi.org/10.3758/s13414-011-0229-0

Freeman, J., \& Simoncelli, E. P. (2011). Metamers of the ventral stream. Nature Neuroscience, 14(9), 1195-1201. https://doi.org/10.1038/nn.2889

Gori, S., \& Facoetti, A. (2015). How the visual aspects can be crucial in reading acquisition: The intriguing case of crowding and developmental dyslexia. Journal of Vision, 15(1), 1-20. https://doi.org/10.1167/15.1.8

Greenwood, J. A., \& Parsons, M. J. (2020). Dissociable effects of visual crowding on the perception of color and motion. Proceedings of the National Academy of Sciences of the United States of America, 117(14), 8196-8202. https://doi.org/10.1073/pnas.1909011117

Harrison, W. J., \& Bex, P. J. (2015). A Unifying Model of Orientation Crowding in Peripheral Vision. Current Biology, 25(24), 3213-3219. https://doi.org/10.1016/j.cub.2015.10.052

He, S., Cavanagh, P., \& Intriligator, J. (1996). Attentional resolution and the locus of visual awareness. Nature, 383(6598), 334-337.

https://doi.org/10.1038/383334a0

Hussain, Z., Webb, B. S., Astle, A. T., \& McGraw, P. V. (2012). Perceptual learning reduces crowding in amblyopia and in the normal periphery. Journal of Neuroscience, 32(2), 474-480.

Intriligator, J., \& Cavanagh, P. (2001). The Spatial Resolution of Visual Attention. Cognitive Psychology, 43, 171-216. https://doi.org/10.1006/cogp.2001.0755

Keshvari, S., \& Rosenholtz, R. (2016). Pooling of continuous features provides a unifying account of crowding. Journal of Vision, 16(3), 1-15.

https://doi.org/10.1167/16.3.39

Kleiner, M., Brainard, D., Pelli, D., Ingling, A., Murray, R., \& Broussard, C. (2007). What's new in Psychtoolbox-3 ? Foreword; - ). What's New. Retrieved from http://www.psychtoolbox.org

Levi, D. M. (2008, February 1). Crowding-An essential bottleneck for object recognition: A mini-review. Vision Research, Vol. 48, pp. 635-654. https://doi.org/10.1016/j.visres.2007.12.009

Mayfrank, L., Kimmig, H., \& Fischer, B. (1987). The role of attention in the preparation of visually guided saccadic eye movements in man. In Eye Movements from Physiology to Cognition (pp. 37-45). https://doi.org/10.1016/b978-0-444-70113-8.50008-4

Motter, B. C., \& Simoni, D. A. (2007). The roles of cortical image separation and size in active visual search performance. Journal of Vision, 7(2), 6-6.

https://doi.org/10.1167/7.2.6

Pelli, D. G. (2008, August 1). Crowding: a cortical constraint on object recognition. 
Current Opinion in Neurobiology, Vol. 18, pp. 445-451.

https://doi.org/10.1016/j.conb.2008.09.008

Pelli, D. G., Palomares, M., \& Majaj, N. J. (2004). Crowding is unlike ordinary masking: Distinguishing feature integration from detection. Journal of Vision, 4(12), 1136-1169. https://doi.org/10.1167/4.12.12

Petrov, Y., \& Meleshkevich, O. (2011a). Asymmetries and idiosyncratic hot spots in crowding. Vision Research, 51(10), 1117-1123.

https://doi.org/10.1016/j.visres.2011.03.001

Petrov, Y., \& Meleshkevich, O. (2011b). Locus of spatial attention determines inward-outward anisotropy in crowding. Journal of Vision, 11(4). https://doi.org/10.1167/11.4.1

Petrov, Y., Popple, A. V., \& McKee, S. P. (2007). Crowding and surround suppression: Not to be confused. Journal of Vision, 7(2). https://doi.org/10.1167/7.2.12

Prinzmetal, W., Presti, D. E., \& Posner, M. I. (1986). Does attention affect visual feature integration? Journal of Experimental Psychology: Human Perception and Performance, 12(3), 361-369. https://doi.org/10.1037/0096-1523.12.3.361

Rosenholtz, R., Yu, D., \& Keshvari, S. (2019). Challenges to pooling models of crowding: Implications for visual mechanisms. Journal of Vision, 19(7), 15. https://doi.org/10.1167/19.7.15

Scolari, M., Kohnen, A., Barton, B., \& Awh, E. (2007). Spatial attention, preview, and popout: Which factors influence critical spacing in crowded displays? Journal of Vision, 7(2). https://doi.org/10.1167/7.2.7

Shechter, A., \& Yashar, A. (2021). Mixture model investigation of the inner-outer asymmetry in visual crowding reveals a heavier weight towards the visual periphery. Scientific Reports, 11(1), 2116. https://doi.org/10.1038/s41598-02181533-9

Song, S., Levi, D. M., \& Pelli, D. G. (2014). A double dissociation of the acuity and crowding limits to letter identification, and the promise of improved visual screening. Journal of Vision, 14(5). https://doi.org/10.1167/14.5.3

Strasburger, H. (2005). Unfocussed spatial attention underlies the crowding effect in indirect form vision. Journal of Vision, 5(11). https://doi.org/10.1167/5.11.8

Strasburger, H. (2020, May 1). Seven Myths on Crowding and Peripheral Vision. IPerception, Vol. 11. https://doi.org/10.1177/2041669520913052

Strasburger, H., \& Malania, M. (2013). Source confusion is a major cause of crowding. Journal of Vision, 13(1), 24-24. https://doi.org/10.1167/13.1.24

Strasburger, H., Rentschler, I., \& Jüttner, M. (2011). Peripheral vision and pattern recognition: A review. Journal of Vision, 11(5). https://doi.org/10.1167/11.5.13

Suchow, J. W., Brady, T. F., Fougnie, D., \& Alvarez, G. A. (2013). Modeling visual working memory with the MemToolbox. Journal of Vision, 13(10). https://doi.org/10.1167/13.10.9

Treisman, A. M., \& Gelade, G. (1980). A feature-integration theory of attention. 
Cognitive Psychology, 12(1), 97-136. https://doi.org/10.1016/00100285(80)90005-5

Treisman, A., \& Schmidt, H. (1982). Illusory conjunctions in the perception of objects. Cognitive Psychology, 14(1), 107-141. https://doi.org/10.1016/00100285(82)90006-8

Tripathy, S. P., \& Cavanagh, P. (2002). The extent of crowding in peripheral vision does not scale with target size. Vision Research, 42(20), 2357-2369. https://doi.org/10.1016/S0042-6989(02)00197-9

Wallace, J. M., Chung, S. T. L., \& Tjan, B. S. (2017). Object crowding in age-related macular degeneration. Journal of Vision, 17(1). https://doi.org/10.1167/17.1.33

Whitney, D., \& Levi, D. M. (2011, April 1). Visual crowding: A fundamental limit on conscious perception and object recognition. Trends in Cognitive Sciences, Vol. 15, pp. 160-168. https://doi.org/10.1016/j.tics.2011.02.005

Yashar, A., Chen, J., \& Carrasco, M. (2015). Rapid and long-lasting reduction of crowding through training. Journal of Vision, 15(10), 1-15.

https://doi.org/10.1167/15.10.15

Yashar, A., Wu, X., Chen, J., \& Carrasco, M. (2019). Crowding and Binding: Not All Feature Dimensions Behave in the Same Way. Psychological Science, 30(10), 1533-1546. https://doi.org/10.1177/0956797619870779

Yeshurun, Y., \& Carrasco, M. (1998). Attention improves or impairs visual performance by enhancing spatial resolution. Nature, 396(6706).

https://doi.org/10.1038/23936

Yeshurun, Y., \& Rashal, E. (2010). Precueing attention to the target location diminishes crowding and reduces the critical distance. Journal of Vision, 10(10). https://doi.org/10.1167/10.10.16

Zhang, W., \& Luck, S. J. (2008). Discrete fixed-resolution representations in visual working memory. Nature, 453(7192), 233-235.

https://doi.org/10.1038/nature06860

Zhu, Z., Fan, Z., \& Fang, F. (2016). Two-stage perceptual learning to break visual crowding. Journal of Vision, 16(6), 16. 


\section{The Role of Transient Attention in Crowding and Feature Binding}

Bahiyya Kewan-Khalayly \& Amit Yashar

\section{Supplementary Information}

\section{Supplementary Analysis Experiment 1}

The results of the 2X4 ANOVA on $\mathrm{Pt}$,

For $\mathrm{Pt}$, cue condition affected performance, $\mathrm{F}(3,45)=7.12, \mathrm{p}=0.001, \eta_{2_{\mathrm{p}}}=0.32$, with means $\mathrm{Pt}: \mathrm{M}=0.76, \mathrm{SE}=0.01, \mathrm{M}=0.79, \mathrm{SE}=0.01, \mathrm{M}=0.76, \mathrm{SE}=0.01$, $\mathrm{M}=0.71, \mathrm{SE}=0.01$, for the neutral, inner, target and outer cue conditions respectively. The effect of cue condition on $\mathrm{Pt}$ interacted with crowding condition, $F(3,45)=7.37, p<0.001, \eta^{2}=0.33$, with larger cue effect in crowded trials compared to the uncrowded trials (Table S2). There was no significant cueing effect or interaction with variability and guessing rate, $p>0.2$. 


\section{ATTENTION ASYMMETRY}

Table S1. Experiment 1 Error distribution. Mean report bias and precision of the error distribution in Experiment 1 (Mean \pm SE).

\begin{tabular}{cccc} 
Display & Cue & Report bias & Precision \\
\hline Neutral & $1.01 \pm .39$ & $.0038 \pm .00064$ \\
Uncrowded & Inner & $1.17 \pm .93$ & $.0038 \pm .00059$ \\
& Target & $.69 \pm .44$ & $.0039 \pm .00061$ \\
& Outer & $.06 \pm .76$ & $.0037 \pm .00057$ \\
& Neutral & $1.59 \pm .9$ & $.00076 \pm .00008$ \\
& Inner & $.92 \pm 1.13$ & $.00093 \pm .00012$ \\
Crowded & Target & $-.42 \pm 1.13$ & $.00081 \pm .00009$ \\
& Outer & $.41 \pm .61$ & $.00076 \pm .00013$
\end{tabular}


Table S2. Experiment 1 Model comparisons. Mean AICc values of the model comparison in Experiment 1 (Mean $\pm \mathrm{SE}$ ).

\begin{tabular}{cccc} 
& \multicolumn{3}{c}{ Model } \\
\cline { 2 - 4 } Cue & Standard model & Two-misreport & One-misreport \\
\hline \hline Neutral & $1131.5 \pm 10.09$ & $1097.59 \pm 11.48$ & $1118.77 \pm 10.43$ \\
Inner & $1125.35 \pm 10.02$ & $1092.74 \pm 10.42$ & $1114.85 \pm 9.63$ \\
Target & $1109.82 \pm 12.35$ & $1084.69 \pm 12.05$ & $1100.72 \pm 12.01$ \\
Outer & $1140.15 \pm 11.35$ & $1092.39 \pm 12.47$ & $1122.35 \pm 10.65$
\end{tabular}




\section{ATTENTION ASYMMETRY}

Table S3. Experiment 1 Best Fitted model parameters. Mean fitted parameters of the standard-mixture model and the two-misreport model in Experiment 1 (Mean \pm SE).

Parameter

\begin{tabular}{|c|c|c|c|c|c|}
\hline Display & Cue & $\gamma$ & $\beta^{I n}$ & $\beta^{\text {Out }}$ & $\sigma$ \\
\hline \multirow[t]{5}{*}{ Uncrowded } & Neutral & $.026 \pm .06$ & -- & -- & $16.15 \pm 1.17$ \\
\hline & Inner & $.033 \pm .07$ & -- & -- & $15.38 \pm 1.21$ \\
\hline & Target & $.033 \pm .07$ & -- & -- & $14.62 \pm 1.02$ \\
\hline & Outer & $.027 \pm .07$ & -- & -- & $15.9 \pm 1.15$ \\
\hline & All & $.03 \pm .016$ & -- & -- & $15.51 \pm 1.09$ \\
\hline \multirow[t]{5}{*}{ Crowded } & Neutral & $.069 \pm .11$ & $.019 \pm .006$ & $.37 \pm .042$ & $18.22 \pm 1.38$ \\
\hline & Inner & $.067 \pm .07$ & $.016 \pm .005$ & $.30 \pm .048$ & $17.88 \pm 1.40$ \\
\hline & Target & $.068 \pm .07$ & $.013 \pm .004$ & $.36 \pm .047$ & $17.82 \pm 1.10$ \\
\hline & Outer- & $.059 \pm .08$ & $.013 \pm .005$ & $.48 \pm .054$ & $19.25 \pm 1.93$ \\
\hline & All & $.066 \pm .014$ & $.015 \pm .003$ & $.38 \pm .043$ & $18.29 \pm 1.3$ \\
\hline \multirow[t]{4}{*}{ All } & Neutral & $.048 \pm .017$ & $.019 \pm .006$ & $.37 \pm .042$ & $17.18 \pm 1.22$ \\
\hline & Inner & $.05 \pm .013$ & $.016 \pm .005$ & $.30 \pm .048$ & $16.63 \pm 1.25$ \\
\hline & Target & $.051 \pm .015$ & $.013 \pm .004$ & $.36 \pm .047$ & $16.22 \pm .96$ \\
\hline & Outer & $.043 \pm .017$ & $.013 \pm .005$ & $.48 \pm .054$ & $17.58 \pm 1.4$ \\
\hline
\end{tabular}


Table S4. Experiment 2 Tilt and Color Error Distribution. Mean report bias and precision for tilt and color in Experiment 2 (Mean $\pm \mathrm{SE}$ ).

\begin{tabular}{|c|c|c|c|c|}
\hline Feat. & Display & Cue & Report bias & Precision \\
\hline \multirow{6}{*}{ Orientation } & \multirow{3}{*}{ Uncrowded } & Neutral & $1.71 \pm .65$ & $2.33 \pm .22$ \\
\hline & & Inner & $1.42 \pm .58$ & $2.42 \pm .18$ \\
\hline & & Outer & $.90 \pm .79$ & $2.26 \pm .16$ \\
\hline & \multirow{3}{*}{ Crowded } & Neutral & $1.83 \pm 1.46$ & $.91 \pm .047$ \\
\hline & & Inner & $2.22 \pm 1.07$ & $.95 \pm .063$ \\
\hline & & Outer & $1.94 \pm 1.32$ & $.90 \pm .047$ \\
\hline \multirow{6}{*}{ Color } & \multirow{3}{*}{ Uncrowded } & Neutral & $-5.38 \pm 1.19$ & $1.65 \pm .080$ \\
\hline & & Inner & $-5.84 \pm 1.29$ & $1.53 \pm .073$ \\
\hline & & Outer & $-4.35 \pm 1.32$ & $1.48 \pm .050$ \\
\hline & \multirow{3}{*}{ Crowded } & Neutral & $-5.06 \pm 1.33$ & $1.31 \pm .072$ \\
\hline & & Inner & $-5.42 \pm 1.12$ & $1.31 \pm .075$ \\
\hline & & Outer & $-4.41 \pm 1.29$ & $1.24 \pm .067$ \\
\hline
\end{tabular}




\section{ATTENTION ASYMMETRY}

Table S5. Experiment 2 Tilt report model comparisons. Mean AICc of the model comparisons in crowded and uncrowded trials in Experiment 2 (Mean $\pm S E$ ). S=Standard, 2M=Two-msreport, $1 \mathrm{M}=$ One-misreport, $\mathrm{SB}=$ Standard with bias, $2 \mathrm{M}=$ Two-misreport with bias, $1 \mathrm{M}=$ =ne-misreport with bias.

\begin{tabular}{|c|c|c|c|c|c|c|c|c|}
\hline \multirow[b]{2}{*}{ Feat. } & \multirow[b]{2}{*}{ Disp. } & \multirow[b]{2}{*}{ Cue } & \multicolumn{6}{|c|}{ Model } \\
\hline & & & $S$ & $2 \mathrm{M}$ & $1 \mathrm{M}$ & SB & $2 \mathrm{MB}$ & $1 \mathrm{MB}$ \\
\hline \multirow{6}{*}{ 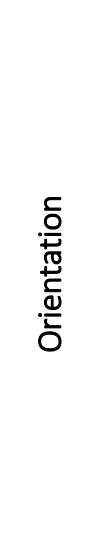 } & \multirow{3}{*}{ 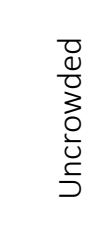 } & Neutral & $848 \pm 15$ & -- & -- & $848 \pm 14$ & -- & -- \\
\hline & & Inner & $2033 \pm 31$ & -- & -- & $1986 \pm 25$ & -- & -- \\
\hline & & Outer & $1992 \pm 26$ & -- & -- & $2032 \pm 31$ & -- & -- \\
\hline & \multirow{3}{*}{  } & Neutral & $1985 \pm 26$ & $1991 \pm 26$ & $844 \pm 16$ & $846 \pm 16$ & $1993 \pm 35$ & $1951 \pm 30$ \\
\hline & & Inner & $1956 \pm 30$ & $1993 \pm 35$ & $1951 \pm 30$ & $1955 \pm 30$ & $857 \pm 15$ & $857 \pm 14$ \\
\hline & & Outer & $2029 \pm 32$ & $1970 \pm 28$ & $1983 \pm 28$ & $2029 \pm 32$ & $1970 \pm 28$ & $1982 \pm 28$ \\
\hline \multirow{7}{*}{ 흥 } & \multirow{3}{*}{ 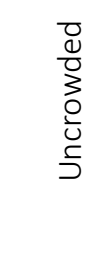 } & Neutral & $934 \pm 13$ & -- & -- & $933 \pm 13$ & -- & -- \\
\hline & & Inner & $1954 \pm 26$ & -- & -- & $1957 \pm 26$ & -- & -- \\
\hline & & Outer & $1955 \pm 26$ & -- & -- & $1951 \pm 26$ & -- & -- \\
\hline & & & & & & & & \\
\hline & & Neutral & $1956 \pm 25$ & $1952 \pm 26$ & $947 \pm 13$ & $946 \pm 12$ & $1941 \pm 27$ & $1943 \pm 27$ \\
\hline & \multirow{2}{*}{$\begin{array}{l}\bar{d} \\
0 \\
0 \\
0 \\
0 \\
0\end{array}$} & Inner & $1942 \pm 27$ & $1938 \pm 27$ & $1943 \pm 26$ & $1939 \pm 27$ & $945 \pm 11$ & $945 \pm 11$ \\
\hline & & Outer & $1964 \pm 25$ & $1966 \pm 24$ & $1964 \pm 24$ & $1961 \pm 25$ & ד & $1962 \pm 24$ \\
\hline
\end{tabular}




\section{ATTENTION ASYMMETRY}

Table S6. Experiment 2 tilt reports model parameters. Mean fitted parameters of the twomisreport with bias model to the tilt reports in Experiment 2 (Mean $\pm S E$ ).

Parameter

\begin{tabular}{lccccccc}
\cline { 2 - 6 } Display & Cue & $\gamma$ & & $\beta^{\text {In }}$ & & $\beta^{\text {Out }}$ & \multicolumn{2}{c}{$\sigma$} & $\mu$ \\
\hline \hline Uncrowded & & & & & & & \\
& Neutral & $.051 \pm .01$ & -- & & -- & $17.45 \pm .85$ & $1.27 \pm .66$ \\
& Inner & $.033 \pm .07$ & -- & & -- & $17.46 \pm .88$ & $1.18 \pm .29$ \\
& Outer & $.059 \pm .01$ & -- & & -- & $17.83 \pm .95$ & $1.56 \pm .61$
\end{tabular}

Crowded

$\begin{array}{llllll}\text { Neutral } & .170 \pm .02 & .069 \pm .013 & .20 \pm .033 & 19.46 \pm 1.22 & 1.84 \pm .74 \\ \text { Inner } & .165 \pm .01 & .070 \pm .011 & .17 \pm .032 & 18.44 \pm 1.08 & 1.41 \pm .62 \\ \text { Outer } & .165 \pm .02 & .048 \pm .011 & .23 \pm .034 & 18.92 \pm 1.11 & 1.39 \pm .68\end{array}$




\section{ATTENTION ASYMMETRY}

Table S7. Experiment 2 color reports model parameters, Mean fitted parameters of the two-misreport with bias model to the color reports in Experiment 2 (Mean $\pm S E$ ).

Parameter

\begin{tabular}{ccccccc}
\multicolumn{1}{c}{ Display } & Cue & $\gamma$ & $\beta^{\text {In }}$ & $\beta^{\text {Out }}$ & $\sigma$ & $\mu$ \\
\hline \hline Uncrowded & & & & & & \\
& Neutral & $0.053 \pm 0.01$ & & & $29.117 \pm 1.91$ & $-4.354 \pm 1.15$ \\
& Inner & $0.090 \pm 0.02$ & & & $28.537 \pm 1.74$ & $-4.431 \pm 0.88$ \\
& Outer & $0.119 \pm 0.02$ & & & $25.286 \pm 1.78$ & $-3.282 \pm 0.87$ \\
Crowded & & & & & \\
& Neutral & $0.113 \pm 0.02$ & $0.021 \pm 0.01$ & $0.007 \pm 0.00$ & $30.784 \pm 1.56$ & $-4.763 \pm 1.06$ \\
& Inner & $0.099 \pm 0.02$ & $0.022 \pm 0.01$ & $0.012 \pm 0.01$ & $30.643 \pm 1.68$ & $-3.617 \pm 1.24$ \\
& Outer & $0.122 \pm 0.02$ & $0.024 \pm 0.01$ & $0.025 \pm 0.01$ & $29.968 \pm 1.37$ & $-3.694 \pm 1.10$
\end{tabular}




\section{ATTENTION ASYMMETRY}

Table S8. Experiment 2's joint distribution in uncrowded trials. Mean fitted parameters of the joint mixture model in uncrowded display (Mean $\pm \mathrm{SE}$ ).

Cue

\begin{tabular}{lccc} 
Parameter & Neutral & Inner & Outer \\
\hline UU & & & \\
Tu & $0.04 \pm(0.04)$ & $0.03 \pm(0.03)$ & $0.04 \pm(0.04)$ \\
UT & $0.01 \pm(0.01)$ & $0.01 \pm(0.01)$ & $0.02 \pm(0.02)$ \\
SDc & $31.16 \pm(31.16)$ & $34.57 \pm(34.57)$ & $33.31 \pm(33.31)$ \\
Sdo & $17.71 \pm(17.71)$ & $17.55 \pm(17.55)$ & $18.11 \pm(18.11)$
\end{tabular}




\section{ATTENTION ASYMMETRY}

Table S9. Experiment 2 joint distribution in crowded trials. Mean fitted parameters of the joint misreport model in crowded trials (Mean $\pm \mathrm{SE}$ ).

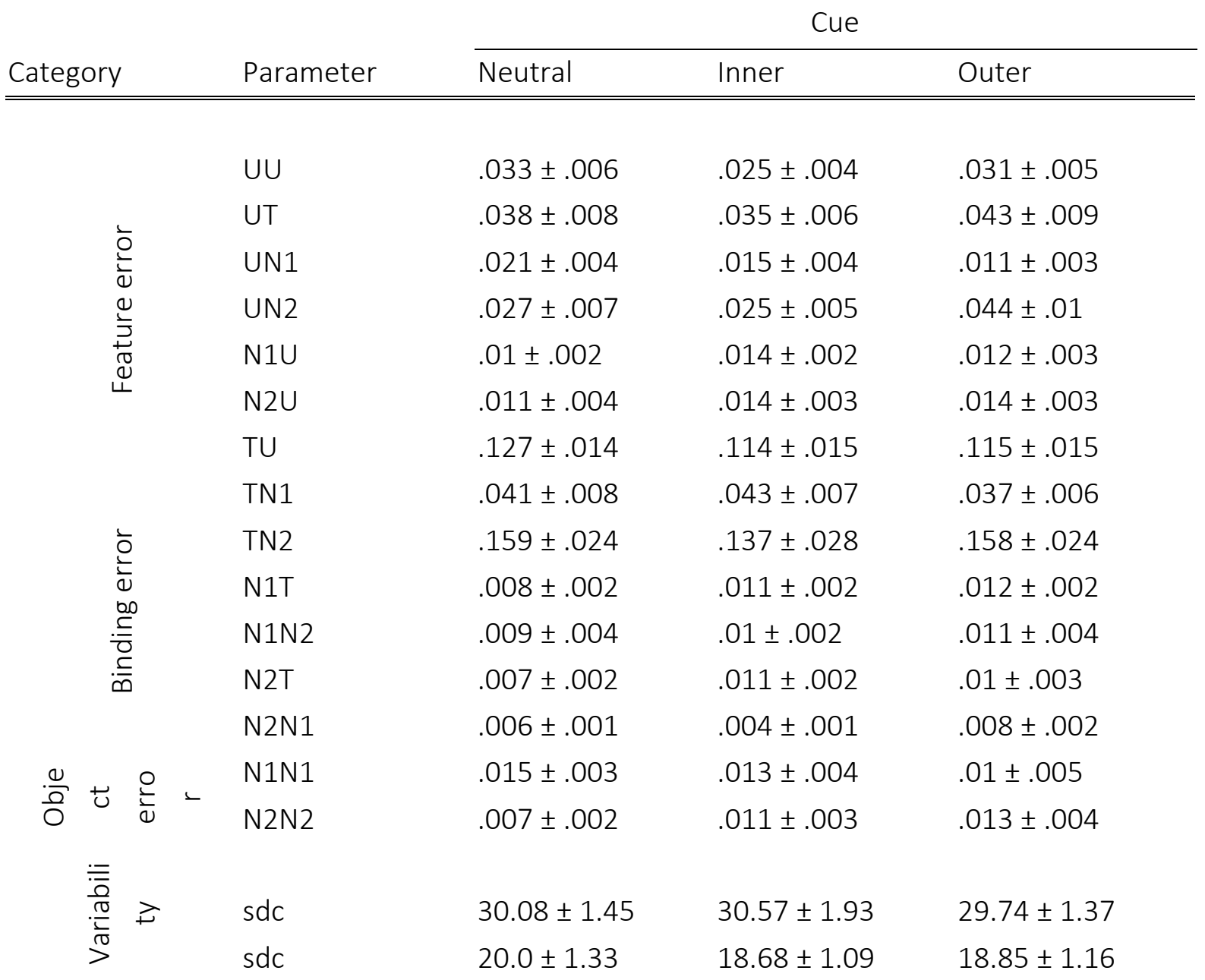

